results obtained on the three steels by the two methods, as follows :

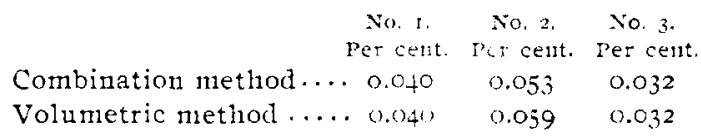

In view of this discrepancy an arsenic determination was made on the No. 2 steel, following exactly the method described in The Chenical Analysis of Iton, p. I88, except that we started with fifty grams. The result obtained was

$$
\text { Arsenic } \ldots \ldots \ldots \ldots \ldots \ldots \ldots \ldots \ldots \ldots \ldots .0 .009
$$

This result seems to indicate that arsenic is the cause of the discrepancy in the No. 2 steel, and apparently confirms again the view that arsenic interferes with the determination of phosphorus by means of molybdic acid.

It is perhaps not essential but may not be amiss to say that where the amount of phosphorus is large a difficulty may arise with the combination method, if ten grams are used to start with, due to the large bulk of molybdenum sulphide obtained. Very large amounts of molybdentum sulphide are difficult to wash clean. Of course the difficulty is easily overcome by starting with less than ten grams.

\title{
ON SOME POINTS IN THE DETERMINATION OF PHOSPHORUS IN STEEL BY THE VOLUMETRIC METHOD.'
}

BY ChAS. B. DUDLEY AND F. N. FEASE, Chemist and Assistant Chemist, Pennsylvania Railroad, Altoona, Pa,

$\bigcirc$

$\mathrm{NE}$ of the most common methods of separating phosphorus from iron, either in ores, pig iron, wrought iron or steel, is by means of molybdic acid, the separation giving rise to the well-known yellow precipitate of ammonium phosphomolybdate. The subsequent treatment of this yellow precipitate is very varied. Some chemists prefer to dissolve the precipitate in ammonia, and then determine the phosphoric acid by means of magnesia mixture. Others prefer to weigh the yellow precipitate just as obtained, and some even to measure the volume of

1 Read at the Baitimore meeting. Decenber 28, Itog3. 
this yellow precipitate in a specially graduated apparatus. Perhaps however, by far the largest number of chenists, prefer to treat the yellow precipitate by some volumetric method, either what may be called the alkali method, described by Handy in the Proceedings of the Engineer's Society of Western Penna., 8, 78 , or the perhaps better known permanganate method, described by Emmerton, published in the Trans. A. I. $M . E ., 15,93$, and subsequently modified by Wood, Shimer. Drown, Jones, and others. We use the permanganate method. This method as we prefer to use it, and with all the precautions which we deem essential to secure uniform and accurate results, except as to the interference produced by arsenic, has been published in the J. Anal. Appl. Chent, 7, 108, and also in the J. Am. Chem. Soc. 15, 519. What follows is a discussion of some points suggested by the work done preliminary to drawing up the method above referred to.

In order that what is given may be clearly understood, it may not be amiss to state that by this method, the steel is dissolved in nitric acid of I.I3 sp. gr., the solution boiled a few seconds. and then treated with potassium permanganate to destroy carbonaceous matter, and possibly secure complete oxidation of the phosphorus; the separated manganese oxide, reduced by a few crumbs of ferrous sulphate, the solution then heated to a definite temperature, and a measured volume of molybdate solution, which is likewise at a definite temperature, added, the vessel enclosed to prevent loss of heat, and shaken vigorously for five minutes, allowed to stand a few minutes for the precipitate to settle, and then filtered. After thorough washing with acid ammonium sulphate wash water, the yellow precipitate is dissolved in ammonia, the solution treated with a definite amount of sulphuric acid, and then passed through the reductor, and finally titrated with potassium permanganate of known strength, the amount of phosphorus being ultinately obtained by multiplying the number of cubic centimeters of permanganate used by the proper factor.

It is obvious that the accuracy of the determination by the method briefly outlined above, depends on several conditions:

I. Is all the phosphorus in the steel taken to start with 
actually in the yellow precipitate obtained, or in other words. can phosphorus be completely separated from iron by nolybalic acid? Tpon this point we have done no work, and cannot express an opinion. The late J. Lawrence Smith used to cham that the complete separation of res small amount of phosphorus from large anounts of iron by means of molyblic aciel was at least coubtful, and that the most reliable procedure consisted in first concentrating the phosphorus into a very small amount of iron, and then separating it from this iron by neans of molybdic acid. Assuming, howerer, that all the phosphorus in the steel is in the yellow precipitate. sereral further questions arise.

II. Is the yellow precipitate of constant composition, that is, is it independent of the conditions under which it in formed? It is obrions that this is a very important point for all those methods which either weigh or measure the yellow precipitate, and also for the permanganate rolumetric method. which in reality actually measures not the phosphorus or phosphoric acid in the yellow precipitate but rather the nolyblic acid, the phonphorus being determined from its relation to the molyblic acich. If now the yellow precipitate is not of constant composition, or more definitely if the relation between the phosphorus and molybdic acid in this precipitate is not a constant one, but is affected by the conditions under which the precipitate is formech. it is clear that these conditions must be definitely defined, and always closely followed, or there will be great uncertainty in the results. What then are the facts in the case? It is well known that almost from the beginning of the use of molybdic acid in phosphorus determinations there has been more or less uncertainty as to the constitution of the yellow precipitate, and especially that, depending on the conditions, it is rery liable to be contaninated with free nolybilic acid. Furthernore, Enmerton has apparently demonstrated in the place cited above, that the temperature of precipitation has a very important influence on the final result. He also clains that the anonnt of free acid in the solution, the concentration of the solution, and the length of time allowed for precipitation also affect the final result. Other workers, especially Wood, in the 
J. Anal. Chem., 1, 138, Shimer, Trans. A. I. M. E., 17, 100, and Drown, Trans. A.I.M.E., 18, 9o, have put a good deal of study on the method. None of the determinations given that we have been able to study, show that the final result is independent of the conditions under which the yellow precipitate is obtained. We ourselves have made a good many experiments, varying the conditions under which the yellow precipitate is obtained, and feel safe in saying that almost any variation in the conditions will produce some difference in the final result. Nuch of our work simply confirms that of other operators. Perhaps it would be worth while to give a single illustration.

Two sets of one gram each of three samples of steel containing different anounts of carbon and phosphorus were dissolved in seventy-five cc. of I.I 3 sp. gr. nitric acid, and further treated exactly alike according to our standard method above referred to, except that before adding the molybdate solution, ten cc. of strong ammonia was added to each flask of one set. This procedure would as is evident, result in the formation of the yellow precipitate in one set in a menstruum containing more ammonium nitrate, and less free nitric acid than the other contained. The results obtained are as follows, the figures being the phosphorus in the three steels:

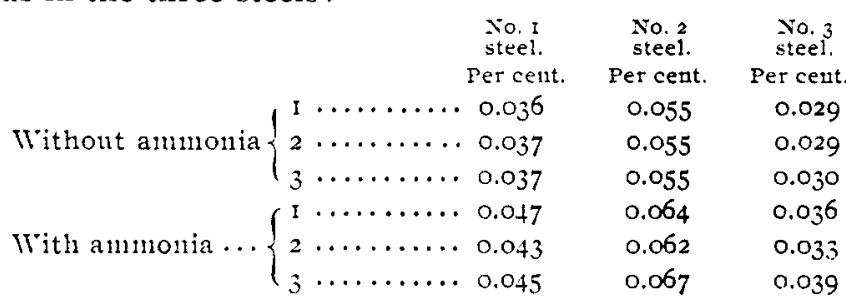

The difference in the results is quite marked. Dimi free acid and increase in annmonium nitrate apparently seads to higher figures and less uniformity in duplicate determinations. Some of our experiments indicate that even a much less change in the proportions of free acid and ammonium nitrate than given above affects the final result. Also that in addition to the variables mentioned above, a different result is obtained when sugar or other organic substance is used to reduce the separated manganese oxide than is given when ferrous sulphate 
is used. Finally there are quite strong indications, that the total amount of molybdic acid present in the solution and eren its tate of oxidation have an influence on the final figures obtained.

If it be allowed then that the conditions, under which the yellow precipitate is formed affect its constitution. or more definitely, affect the ratio between the molybdenum and phosphorus in this precipitate. or what amounts to the same thing. affect the final result, two things are evident.

I. In order to secure accurate and concordant results, it is necessary that the conditions uncler which the yellow precipitate is obtained shall be very clearly and sharply defined, and that on making the analyses, these conditions shall be very rigidly maintained.

2. It will not do to use ratios based on the analysis of the Ellow precipitate obtained under one set of conditions as a means of calculation for precipitates obtained uncer another and aifferent set of conditions.

As an example of this second statentent, it seems clear, if the preceling riews are correct. that if a chemist obtains a quantity of the yellow precipitate from sodium phosphate with no iron present, and the conditions entirely different from those which prevail in the determination of phosphorus in steel, and then makes an analysis of this yellow precipitate to determine the ratio between molybdic acid and phosphorus, although the figures he obtains may be quite accurate, he is still not at liberty to use these figures in determining phosphorus in iron and steel and claim that they give accurate results. It is of course conceded that they may be accurate but there is no certainty of this. It should be stated that the amount of phosphorus in the yellow precipitate is so small, and the anount of nolybdic acid is so large, that there is room for considerable rariation in ratios without giving rise to very serious differences in results, especially in steels low in phosphorus. This fact may help to account for the claim so generally made by different chemists that their results agree within reasonable limits of error with those of other good chemists. But if it is desired to obtain fairly accurate and concordant results, we see no way of escaping from the two statements given above. 
If these points be conceded it is obvious that a still further very important question arises, viz.:

III. How shall the proper factor for use with the permanganate of potash be obtained? It is clear, since the permanganate of potash solution is standardized against metallic iron. that we need two ratios, first, the ratio between molybdic acid and iron, and second, the ratio between molybdic acid and phosphorus in the yellow precipitate obtained under definite conditions. What then are these ratios? On looking over the literature on the subject, we find two or three serious uncertainties. The ratio between iron and molybdic acid is eridently affected by the change which takes place in the molybdic acid when it passes through the reductor, and we do not find that there is agreement between those who have worked on this point. Furthermore, Jones has apparently shown in the Trans. A. I. M.E. 18, 705 , that the use of the reductor gives different results than are obtained when the molybdic acid in the yellow precipitate is reduced by granulated zinc in a beaker as originally. proposed by Emmerton. Again the published ratios between molybdic acid and phosphorus, vary between 100 : I.54 and 100 : I.90 if we may use our own figures for illustration. It is clear therefore that there are two difficulties in the way of using any of the published ratios, first, which ratios shall be used, and second, the point already discussed quite at length, that these ratios vary, or at least the final results vary according to the conditions under which the yellow precipitate is obtained.

Two methods of procedure are clearly open to us.

First, obtain a quantity of the yellow precipitate under the conditions which we deem essential, and then make independent determinations of the phosphorus and molybdic acid in this precipitate. This would give the ratio between these two constituents. Then pass a quantity of the same precipitate through the reductor and titrate with permanganate. This will give the ratio between molybdic acid and iron. This we have not done yet, partly for lack of time and partly from the difficulties of the analysis. In our experience it is not easy to dry and weigh the yellow precipitate without its undergoing change in the process, and our experience with methods of determining 
molybdic acid is also meager. There is need of sone good and careful work on this point.

In view of these uncertainties, we chose the other method of procedure, viz.:

Second, determine the phosphorus in several samples of steel by the most accurate known grarimetric method, then make an analysis for phosphorus in these same steels by the volumetric method, and then use such a factor with the potassium permanganate as will bring these results together. The work cone on the steels chosen for this purpose is given in our paper "On an Attempt to Find the Amount of Phosphorus in Three Samples of Steel."

It is evident that this procedure, while it enables us to get a factor to use with the potassium permanganate in the phosploorus determinations by the rolumetric method as we use it, does not throw any light on the ratios between iron and nolybdic acid, and between molybdic acid and phosphorus in the yellow precipitate as we obtain it. But if we assume that the ratio of iron to molybdic acid is $100: 90.76$ it follows that the ratio of molybdic acid to phosthorus in the yellow precipitate as we obtain it is IOO: 1.90 and this is the calculation which we have used in our published volumetric method above referred to.

One further question may arise, aiz.: In view of the apparent variability of the yellow precipitate according to the conditions under which it is obtained,

IV. If the conditions are made constant, will the results obtained be uniform and agree with each other?

In reply to this we may say that so far as our experience has gone, the agreement between duplicate deterninations on the same sample of steel where the conditions of the method as published are rigidly adhered to, rarely exceeds more than a couple of thousandths of a per cent., also lifferent operators using the published method on the same sample of steel, after a little experience is obtained with it, rarely disagree more than three or four thousandths. 